\title{
Development of large-diameter flat mesh-lenses for millimetre wave instrumentation
}

Giampaolo Pisano, Alexey Shitvov, Paul Moseley, Carole Tucker, Giorgio Savini, et al.

Giampaolo Pisano, Alexey Shitvov, Paul Moseley, Carole Tucker, Giorgio Savini, Peter Ade, "Development of large-diameter flat mesh-lenses for millimetre wave instrumentation," Proc. SPIE 10708, Millimeter, Submillimeter, and Far-Infrared Detectors and Instrumentation for Astronomy IX, 107080D (9 July 2018); doi: 10.1117/12.2314063

Event: SPIE Astronomical Telescopes + Instrumentation, 2018, Austin, Texas, United States 


\title{
Development of large-diameter flat mesh-lenses for millimetre wave instrumentation
}

\author{
Giampaolo Pisano*a, Alexey Shitvov ${ }^{\mathrm{a}}$, Paul Moseley ${ }^{\mathrm{a}}$, Carole Tucker $^{\mathrm{a}}$, Giorgio Savini ${ }^{\mathrm{b}}$, Peter Ade $^{\mathrm{a}}$ \\ aSchool of Physics and Astronomy, Cardiff University, Queen's Building, The Parade, CF24 3AA \\ Cardiff (United Kingdom); ${ }^{\mathrm{b} O p t i c a l ~ S c i e n c e ~ L a b o r a t o r y, ~ P h y s i c s ~ a n d ~ A s t r o n o m y ~ D e p a r t m e n t, ~}$ \\ University College London, Gower Street, London WC1E 6BT
}

\begin{abstract}
We present novel optics solutions based on metal mesh flat lenses which can be used to mimic a refracting lens. This approach removes the bulk of the dielectric materials (polyethylene or silicon) required for fast optical refracting systems. Additional attractive property of these lenses is that they filter out unwanted higher frequency radiation, they are easy to anti-reflection coat and they are extremely light. Measurements from $300 \mathrm{~mm}$ diameter prototypes of both a Fresnel-type graded-index lens and a phase-delay type mesh-lens are presented.
\end{abstract}

Keywords: Cosmic Microwave Background, Optics, Polarimetry, Millimeter-wave instrumentation.

\section{INTRODUCTION}

The progress in the field of millimeter and sub-millimeter astronomy often implies the development of instruments with larger and larger diameters. In particular, in the field of Cosmic Microwave Background radiation, the detection of the polarized B-modes puts challenging requirements on the sensitivities of these instruments. This results in having quasioptical systems with large throughputs requiring highly convergent beams, i.e. short focal lengths.

Some of these quasi-optical systems utilize lens-based designs. Lenses can be made with low refractive index material, such as polypropylene or polyethylene, or with higher refractive index materials such as silicon or alumina. In either case, the required diameters are of the order of $50 \mathrm{~cm}$. In the case of low refractive index materials, the required focusing power is achieved by designing very thick and bulky lenses leading to undesirable mass. Although the reflection losses can be minimized with the application of specific anti-reflection coating layers, there are still large losses associated with the thick non-ideal lossy medium. Alternatively, thinner lenses can be designed with higher refractive index materials, however the design and the implementation of the anti-reflection coatings is more challenging because of the larger mismatch existing between the medium and free-space. In addition, the focal planes of modern CMB instruments typically accommodate multichroic detectors which requires the optical system to operate across very large bandwidths.

Here we present alternative approaches to designing large diameter millimeter-wave lenses, which rely on the use of metamaterials. Two types of metamaterial lenses have been developed using the well-known mesh-filter technology [1, 2]: a graded-index device (GrIn-lens) [3] and a device based on a phase-delay approach (mesh-lens) [4]. These components are flat and very thin compared to the classical dielectric lenses. These designs have been realized with 5$10 \mathrm{~cm}$ diameters using the same well-established manufacture techniques and have been successfully tested at millimeter wavelengths [3, 4]. Given the present instrumentation requirements, we are now pushing the above technologies to large diameters. This is particularly challenging because this development is not a simple scaling of the previous realizations.

We have recently developed a large diameter $(30 \mathrm{~cm})$ graded-index lens using a Fresnel-type design [5]. This lens, just $2 \mathrm{~mm}$ thick, was proven to work at specific frequencies. It will require further developments in order to improve the reflection losses and the phase behavior across large frequency bands.

In this work we report on new experimental results of the GrIn lens and, in more details, on the development of a large diameter $(30 \mathrm{~cm})$ mesh-lens. We report on the preliminary test results of the beams produced by the large-diameter mesh-lens together with the predicted performance.

*giampaolo.pisano@astro.cf.ac.uk; phone ++44(0)29208 75314.

Millimeter, Submillimeter, and Far-Infrared Detectors and Instrumentation for Astronomy IX, edited by Jonas Zmuidzinas, Jian-Rong Gao, Proc. of SPIE Vol. 10708, 107080D

(c) 2018 SPIE · CCC code: 0277-786X/18/\$18 · doi: 10.1117/12.2314063 


\section{MESH FILTERS TECHNOLOGY}

The mesh-technology had a huge growth in the last decades in association to the development of filters for millimeter wave astronomical instrumentation. The original mesh filters where 'air-gap' type filters, i.e. made with free-standing metal grids sometimes supported by thin polymer substrates. The grids were stacked together and held at specific distances with spacing rings. Although these multi-layer air-gap filters have very good performance and low losses, they are very fragile and cannot be easily produced in large diameters. Later on a new technique was developed by the authors of this work which enabled the grids to be hot-pressed within polypropylene layer and be embedded into a single polymer slab. These 'hot-pressed' type filters are very robust, still relatively low-loss, can work cryogenically, can be machined to any profile and can be produced in large diameters. For more details about these techniques see [1, 2].

In the last decade the mesh-filter technology had further developments in terms of design and applications. Anisotropic grids have been employed to mimic birefringent materials and to realize large bandwidth retarders such as mesh HalfWave Plates (HWPs) [6] and reflective HWPs [7]. These HWPs have found application in astronomical polarimetry where the instruments are required to rotate and modulate the incoming linear polarization.

More recently, close packed metal meshes have been used to synthesize artificial dielectric materials [8] and inhomogeneous grids have been designed to manipulate the phase across surfaces. These new two approaches allowed to develop large diameter flat lenses based on the mesh technology: GrIn-lenes [3] and mesh-lenses [4]. GrIn lenses are based on graded-index artificial dielectrics. New developments and results about this technology will be discussed in Sec.3. Mesh lenses are based on highly transmissive mesh filters with variable phase delays. In Sec.4 and the following we are presenting the results of the first prototype of a large diameter mesh-lens.

\section{NEW RESULTS ON LARGE DIAMETER GRADED INDEX FRESNEL LENSES}

Modelling of thin metal meshes with set complex impedance values based on the mesh geometry, led to the initial concept [8] that a homogeneous stack of metal mesh layers would exhibit the behaviour of an effective medium with a given refractive index at wavelengths greater than that of the mesh resonance behaviour. This initial work [8] explored (through FEA simulations) the variations in index that could be produced by varying the size of a capacitive square patch as well as the fixed separation between layers and the number of layers. These findings also hinted at a dependence with frequency which is much weaker than that typically exhibited by resonant mesh behaviour and, unlike some other metamaterial structures that demonstrate more exotic refractive index values, such as negative index [9], this approach was shown to be valid over a large frequency range.

As the optical parameters derive from the local distribution of mesh patterns, local variation of the effective refractive index could be achieved through gradual variation of the mesh parameters. This was achieved through the design and test of a graded index lens [3]. This first design was a 70mm diameter, F3.5 lens that operated from 100 to $320 \mathrm{GHz}$. The graded index profile of the lens was based on the Woods formalism, which meant that the lens required an index range of $2.70-1.49$ achieved with 20 equi-spaced layers for an overall thickness of $2 \mathrm{~mm}$. From the original and subsequent publications [10] it was shown that the performance is close to that of an ideal lens.

The flexibility of this effective index approach combined with the strong/weak coupling of the index value to the lengths of the square (now rectangles) on the two axes parallel and perpendicular to the field excitation, allowed for the creation of a polarisation-splitting lens [11]. This device has a by-design bi-refringence which is distributed to allow two semiindependent lenses to co-exist in the same physical space focusing an unpolarised source into two spots 10mm apart on the same focal plane. Each spot containing the orthogonal linear polarisations components of the source.

In both cases these designs have a significant insertion loss, due to the transition from vacuum (air) to a high (higher than the supporting polymer substrate) refractive index, so a custom ARC coating is required. However, because traditional graded index theory can be used, design iterations can be performed quickly.

\subsection{Design}

Scaling up the $70 \mathrm{~mm}$ lens to $300 \mathrm{~mm}$ and above is not practical using the direct Woods formalism. To achieve such a large diameter, either the central refractive index would have to be increased to excessively high values (lowering the capacitive cut-off frequency) or the overall thickness of the lens would need to be increased, with associated increase in the number of mesh layers. Therefore in order to maintain similar mesh parameters as for the previous lenses, a Fresnel 
graded index profile was used. This replicates the same process used to design a traditional Fresnel lens, replacing concentric rings of increasing curvature, with increased variation of the index gradient. This means that the overall index range is compressed to values that are more easily obtainable. Figure 1 shows the refractive index profile of a $300 \mathrm{~mm}$ Fresnel lens with a focal length of $1050 \mathrm{~mm}$, optimized for $150 \mathrm{GHz}$. The variation in index is achieved by applying a radial profile to the size of square copper patches, then 20 of these identical mesh layers are combined, separated by $100 \mu \mathrm{m}$ polypropylene, yielding a total thickness of $2 \mathrm{~mm}$. The centre index of 2.7 was chosen so that the previous metamaterial parameter space from the last lens can be used, thus limiting the number of free parameters.

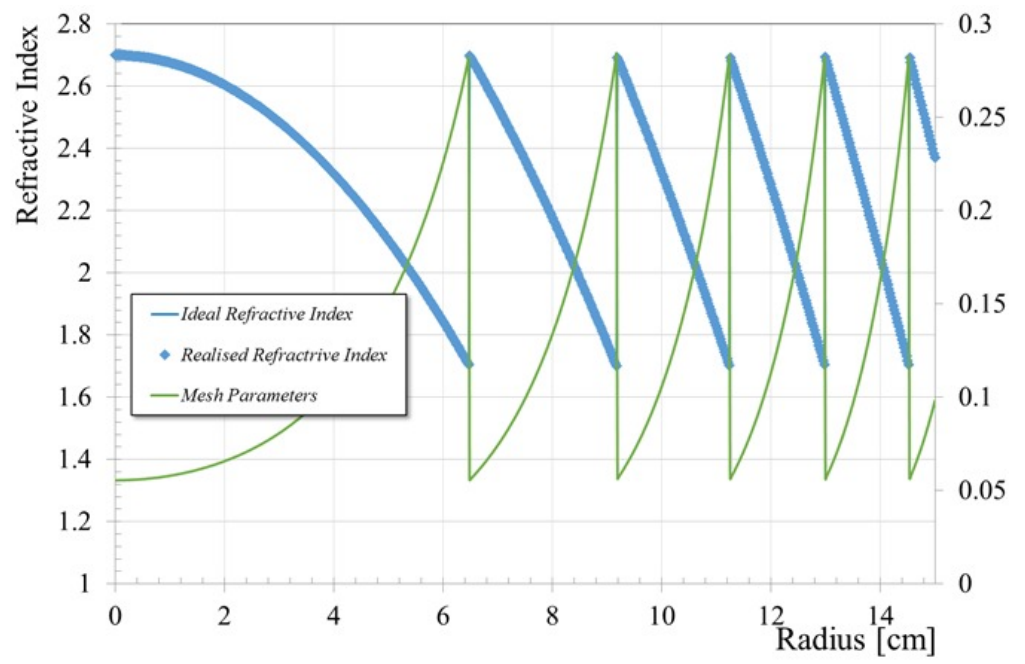

Figure 1. The radial refractive index distribution of the Fresnel lens (blue curve) and the corresponding mesh parameters (green curve).

The lens was fabricated using the hot press technique described in section 5 to produce a solid robust device which is shown in Figure 2.

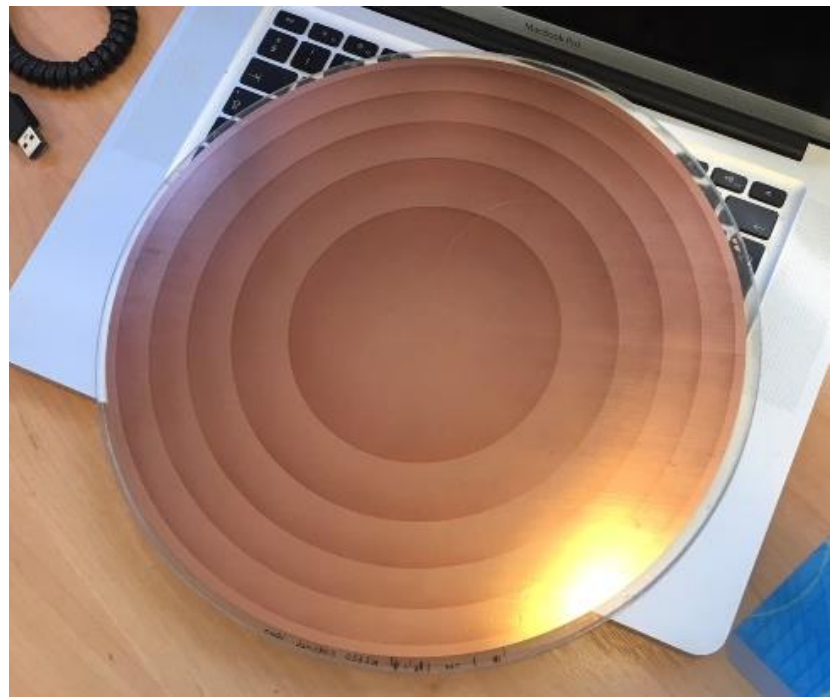

Figure 2. The completed 300mm metal mesh Fresnel lens. 


\subsection{Experimental results}

Using the same beam scanning configuration described in section 6.1 the lens was characterised to see how its performance compares to an ideal lens. The central design frequency of the lens is $150 \mathrm{GHz}$, but to verify its operation over a broad frequency range, it was measured over the bands $110-170 \mathrm{GHz}$ and $222-320 \mathrm{GHz}$ (corresponding to the available VNA frequencies).

A 2D scan performed on a symmetrical plane and in close proximity of the predicted focal region of the lens $(\mathrm{z}=$ $1050 \mathrm{~mm}$ ) was performed showing a clear focus for all frequencies. One example of such a 2D beam cut is shown in Figure 3. We found that while the lens does focus at the predicted focal length the latter does change linearly with frequency as shown in Figure 4. The focal length frequency dependence exhibits the typical Fresnel lens behaviour showing similar focal lengths at multiples of the same frequency, behaviour which is not due to the underlying metamaterial structure.

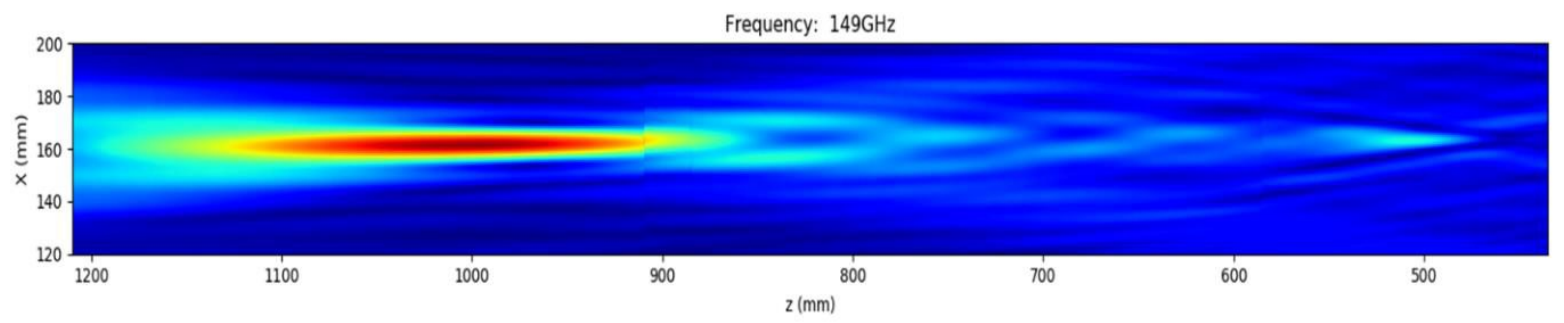

Figure 3. An example of a measured $2 \mathrm{~d}$ beam cut of the lens. A clear focal region can be seen.
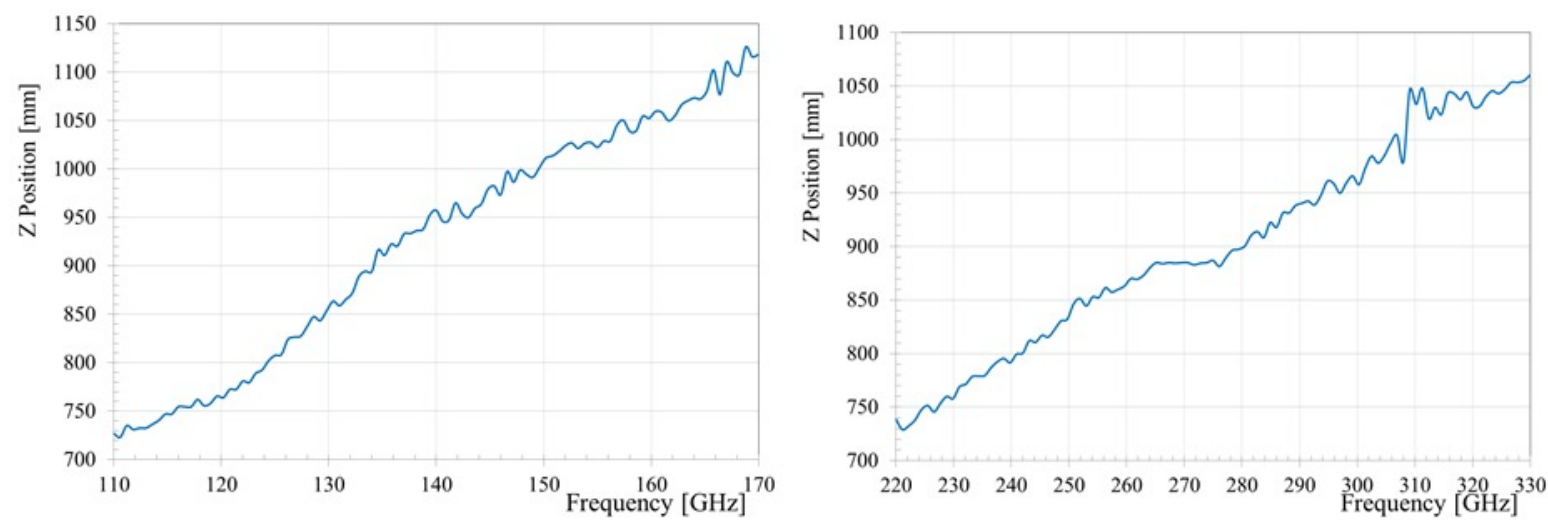

Figure 4. The optimum focus of the lens is plotted for each frequency. It should be noted that b) is double the frequency of a), yet they both have a similar focus behaviour.

At each optimal focus position an X-cut was taken and compared with the Airy fit for an ideal lens with equivalent focal length. It can be seen from figure 5 that there is very good agreement with the model around the design frequency and that good agreement is maintained for higher frequencies.
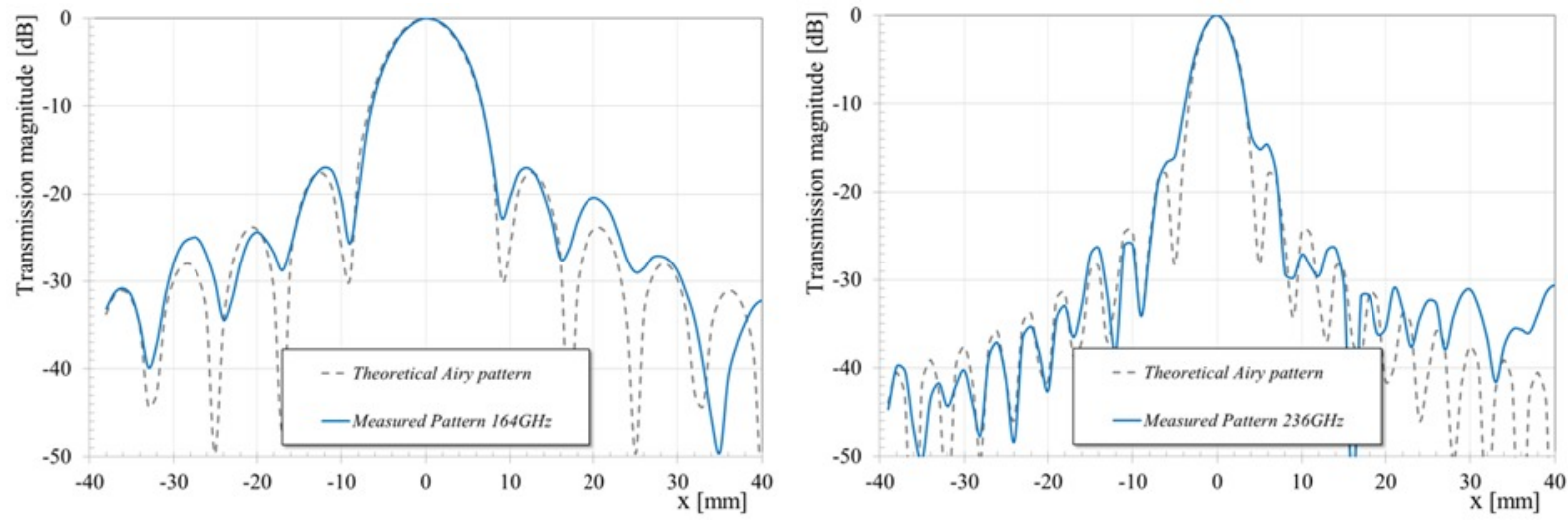

Figure 5. Comparison of measured beam cuts vs the ideal Airy model of a lens at a) $164 \mathrm{GHz}$ and b) $236 \mathrm{GHz}$ 


\section{LARGE DIAMETER MESH LENS DESIGN}

\subsection{Working principle}

The mesh-lens working principle is based on the direct manipulation of the phase-front of a beam [4]. A plane wave is converted into a spherical wave front and vice versa. This is achieved by introducing local phase delays across the beam by means of dielectrically embedded multi-grid structures similar to those used in mesh filters. The difference is that the grids are now inhomogeneous, i.e. their geometry varies across their surfaces. When the different inhomogeneous grids are aligned and stacked together they form a two-dimensional array of pixels. Each pixel is a column of aligned squares with variable size which are suspended within the dielectric material. Each pixel is designed and optimized separately, not only to provide high transmission but also to introduce the specific phase delay which would be required by the equivalent classical lens in that specific location.

\subsection{Design}

The goal is to design a $30 \mathrm{~cm}$ diameter mesh-lens, with an $f$-number $f / 4$ (focal length $f=1200 \mathrm{~mm}$ ), working in the frequency range $75-175 \mathrm{GHz}$, i.e. within a bandwidth $2.3: 1$ or an $80 \%$ relative bandwidth. These requirements can be met using a stack of 24 polypropylene embedded capacitive grids with one anti-reflection coating layer on each side of the lens. The polypropylene (PP) refractive index is assumed to be $n_{\mathrm{PP}}=1.50$ and that of the anti-reflection coating, which is porous PTFE, to be $n_{\mathrm{ARC}}=1.25$. The grid period $(g=300 \mu \mathrm{m})$ is the same across the grid surfaces and between all the grids; what is changing is the size of the suspended squares within each pixel cell.

The transmission and the phase delay of each pixel are modelled using the propagation matrix formalism. The reflections between all the polypropylene embedded grids and their match with free space is automatically taken into account. The grids are considered to be two-dimensional with zero thickness and modelled as transmission line shunt elements. Their complex admittances, when embedded in PP, are accurately calculated using finite-element commercial software (Ansys HFSS [12]). The conductive losses of the grids are automatically taken into account because they emerge from the real part of the admittances.

The propagation matrix code optimizes one eighth of the total number of pixels because these solutions will be then repeated 8 times around the optical axis (see inset in Fig.6). However, although only 12.5\% of the total, this number is still very large. In previously realized devices, with diameters around $\sim 50 \mathrm{~mm}$, this number was of the order of one thousand. In this design the number exceeds one hundred thousand. In addition, the number of grids has more than doubled ( 24 vs 10 ) making the whole design computationally heavy with a normal PC. For the above reasons the propagation matrix code had different optimization and interpolation procedures in order to deal with this large number of pixels.

The target of the overall optimization was set to be the conversion of a flat phase beam, from one side of the lens, into a spherical phase front, on the other side of the lens. The outgoing radiation would then ideally converge at the focal point $f$. All the phase delays are calculated relative to the principal pixel, chosen to be the one in the middle of the lens. The mesh lens grids had a symmetric design, i.e. there were $12+12$ grids mirrored about the centre plane. The grids were built pixel by pixel from the centre to the edge of the lens and, depending on the distance from the centre, each pixel was optimized to provide a specific frequency-dependent phase delay while maximizing its transmission. Fig. 6 shows as example how the pixel geometry of the first three grids (out of 12) evolves from the center to the edge of the lens. The pixels are sorted and numbered as a function of their distance from the center. The parameter $b$ is the size of the square and $g$ the period of the cell. A ratio $b / g \sim 1$ means a square almost filling the cell, while $b / g \sim 0$ means a very small square. It can be seen how these parameters evolve in a complex fashion in order to satisfy the transmission and phase requirements of the target lens. Note also a sudden jump, around the $70000^{\text {th }}$ pixel, which meant a complete change in the filter 'recipe' required to achieve the larger phase-shift at those radial distances. 


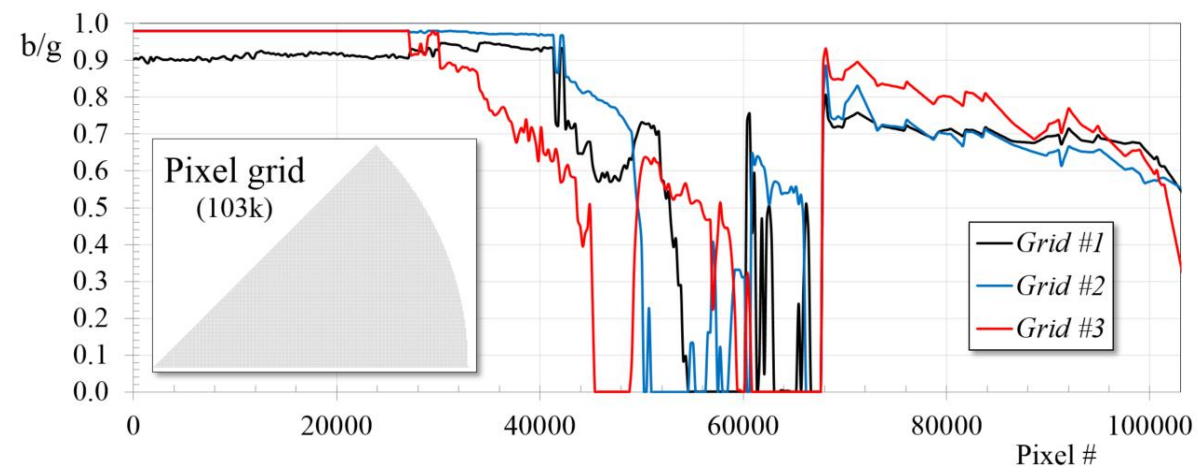

Figure 6. Examples of grid designs. For each pixel of the first three grids, the square size $b$ normalized to the cell period $g$ is plotted as a function of radial distance from the centre. Inset: section of pixels of the mesh lens optimized by the propagation matrix code which, for a $30 \mathrm{~cm}$ diameter lens, comprises $\sim 103 \mathrm{k}$ pixels.

The expected performance of each pixel can be quantified by calculating its transmission and its phase error, integrated across the frequency of operation $(75-175 \mathrm{GHz})$. The phase error is the difference between the phase provided by the propagation matrix code and that expected by the ideal lens at that specific location. The averaged pixel transmissions and phase errors as a function of their radial distance from the centre are reported Fig.7. The overall performance of the lens can be calculated at a first order by averaging the transmissions and the phase errors of all the pixels. This leads to average transmission and phase errors equal to 0.933 and 22.9 degrees respectively.

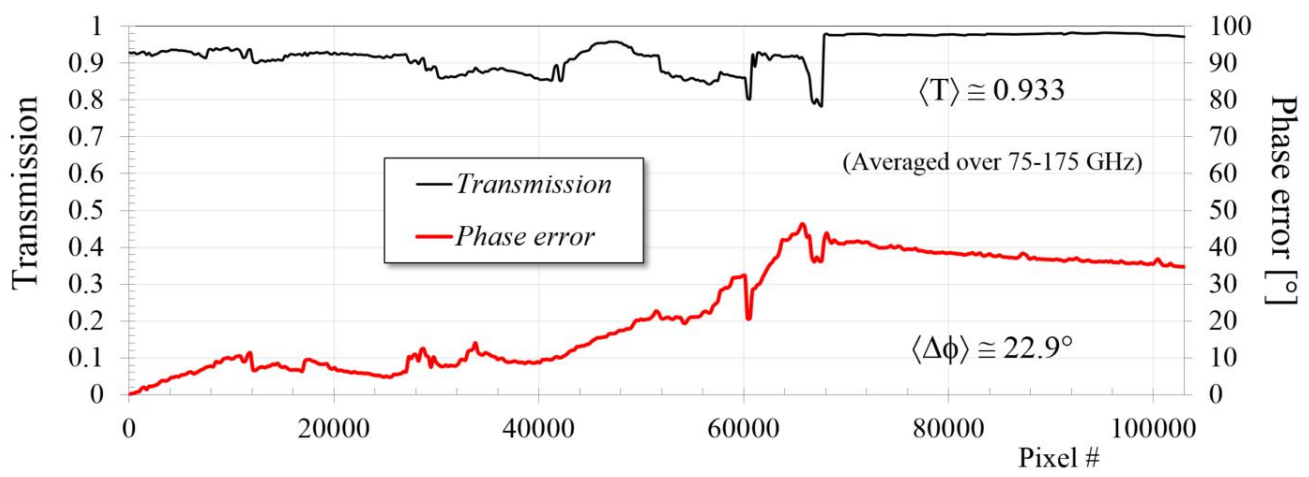

Figure 7. Pixel transmissions and phase errors, averaged over the frequency range $75-175 \mathrm{GHz}$, sorted as their radial distance from the center.

\subsection{Finite element modelling}

Although finite-element analysis was initially used to extract the complex admittances of the grids, it was not possible to run a finite-element model of the final lens design and check its performance. The model would have been too large because it would require a spherical wave interacting with $\sim 19.2$ million square embedded elements. However, it was possible to run a check by modelling just a 'slice' of the lens. The slice was a one-pixel row starting from the central pixel (half of it) and included 512 pixels out to the edge of the lens (see Fig.8a). By introducing symmetry planes and periodic boundaries across the cuts, it was possible to model a cylindrical lens with the same focusing power of the mesh lens, but based only on a fraction of its original pixels.

Fig. $8 \mathrm{~b}$ shows a $90 \mathrm{GHz}$ incident wave generated by a Gaussian beam with its beam waist located at the focus of the lens (not included yet in the model). In Fig.8c it can be seen how, by introducing the mesh lens into the model, the spherical 
wavefront is almost fully converted into a plane wavefront. Some diffraction is occurring around $4 / 5$ of the radius. This is attributable to the sudden change in the pixel 'recipes', already mentioned earlier, and visible on the right of Fig.8a. The results of these preliminary simulations are the first indication of the effective focusing power of the mesh lens design.

a)

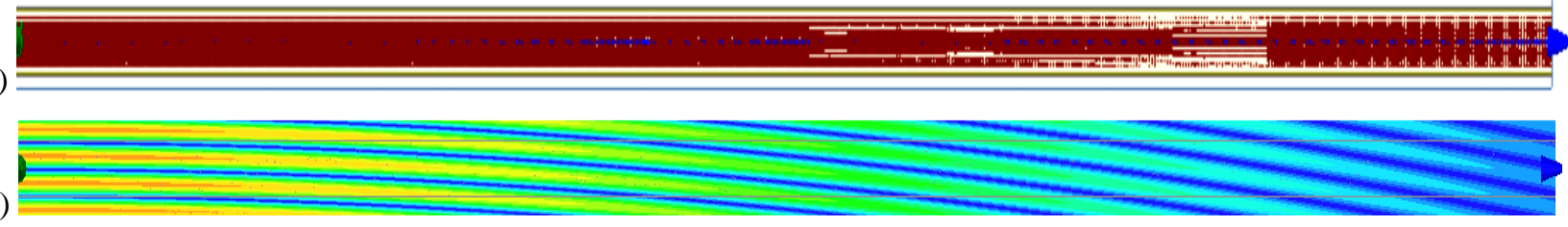

c)

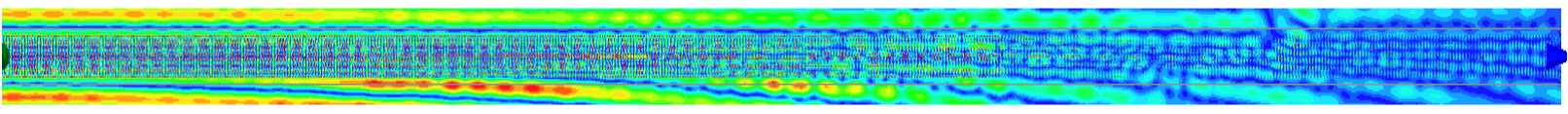

Figure 8. a) HFSS model of a one-pixel row of the mesh lens, equivalent to a cylindrical lens. b) Spherical wavefront generated by a Gaussian beam located at the focus of the lens. c) Conversion of the spherical wavefront into planar wavefront operation by the introduction of the mesh-lens $(90 \mathrm{GHz})$. All the pictures have been expanded along the vertical axis for better clarity.

\section{MESH LENS MANUFACTURE}

The multi-layer lens was manufactured using standard Cardiff University metal mesh-technology manufacture techniques. The device consisted of 24 active layers of photo-lithographically patterned $\mathrm{Cu}$ capacitive structures, precisely spaced and stacked using polypropylene substrates. The production of the photolithographic masks required 2D CAD files which, given the complexity of the geometries, could only be generated by a dedicated code based on the results of the propagation matrix code to give a standard large dxf type file.

There was a high demand for precision alignment of the layers, ideally at the pixel level although there is some tolerance to this requirement (post-manufacture measurement with an optical microscope suggested that the alignment achieved was as good as \pm 1 pixel). The layered assembly was fused together using the Cardiff hot-pressing technique, was checked for pattern uniformity and alignment and then a final ARC layer was applied using an LDPE gluing layer. The unmounted final device is shown in Figure 9.

Note that the total lens thickness is $3642 \mu \mathrm{m}$ and its weight less than $300 \mathrm{~g}$, both quantities much smaller than those of a dielectric lens built to the same diameter and focal length.
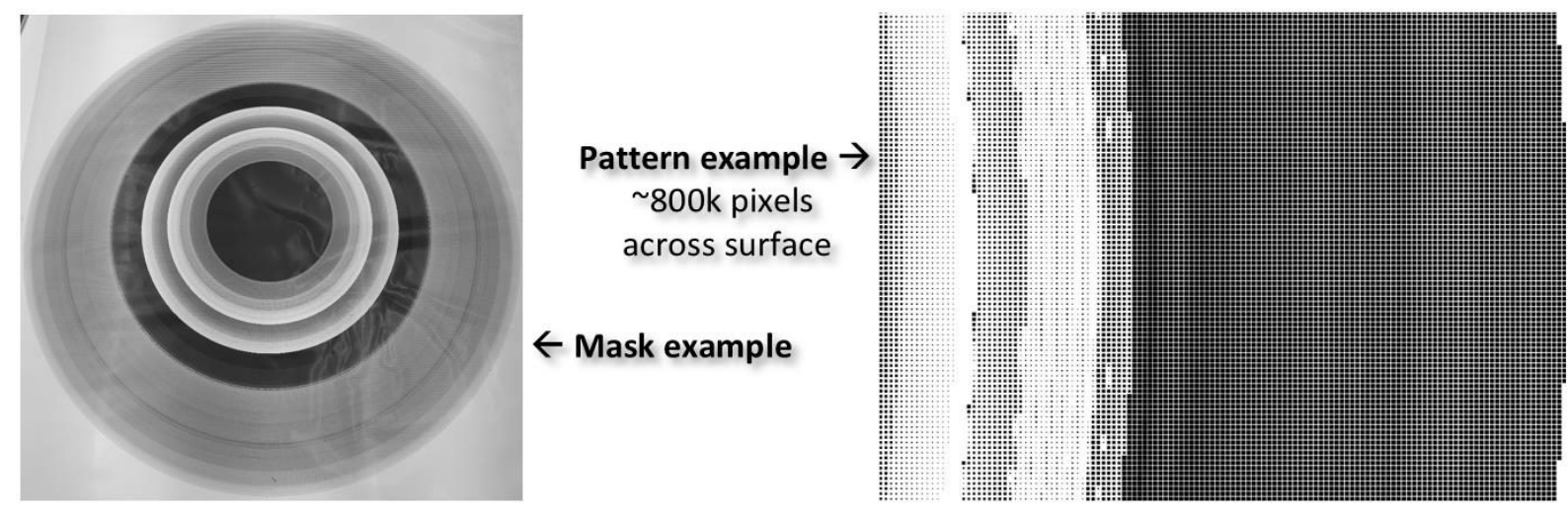

Figure 9. Example of one of the 12 acetate masks used for the photolithographic processing of the 24 single grids making up the lens. 

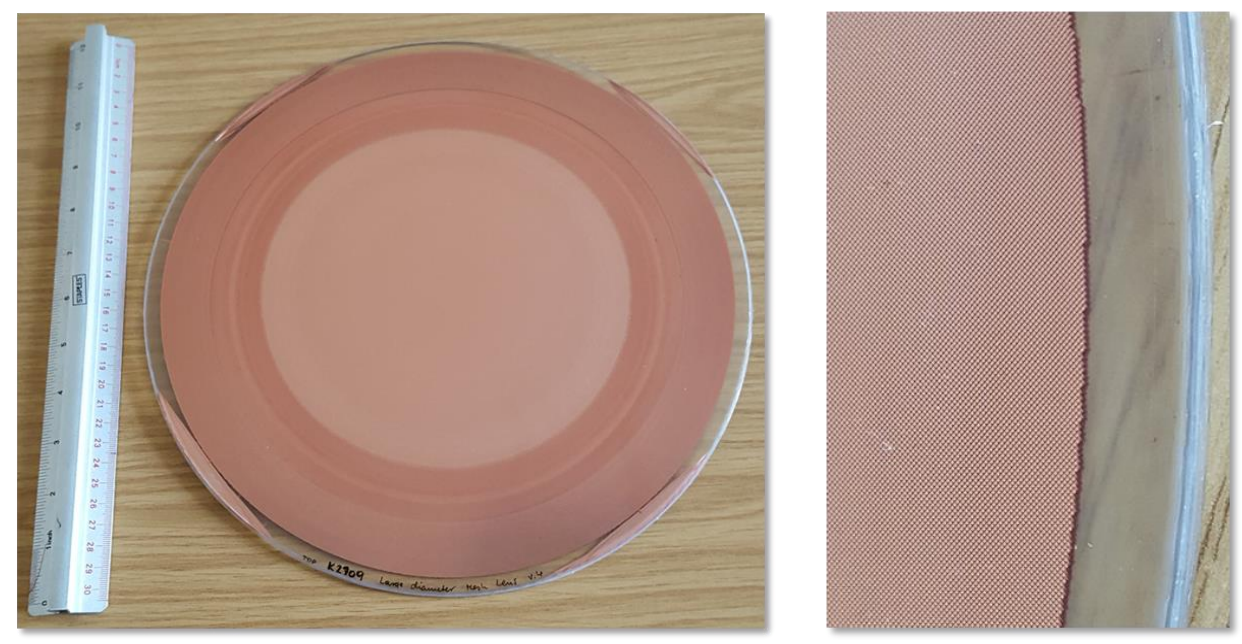

Figure 10. Image of the large diameter mesh-lens prototype with a zoom-out of the grid detail at the edge of the device. This photograph was taken before the lens was AR-coated (as the ARC material is optically opaque).

\section{MESH LENS EXPERIMENTAL CHARACTERISATION}

\subsection{Experiment Setup}

The experimental characterization of the mesh lens prototype was carried out using a three-axis near-field probing test bench, see Fig. 11. The transmission magnitude and phase measurements were taken in the frequency range 110$170 \mathrm{GHz}$ using a commercial Rohde \& Schwarz ZVA 67 analyzer equipped with ZVA-ZC170 frequency converters. The VNA readout, the electronic controls of the three linear stages and the post-processing of the measured data were automated with the aid of the National Instruments LabView software and Python scripts, running on a personal computer. The transmitter power head was fitted with a standard $20 \mathrm{~dB}$ rectangular horn antenna (Flann Microwaves 29240-20). The signal travelling through the lens was detected with a scanning open-ended rectangular waveguide probe (standard WM-1651). In order to minimize standing waves, the probe was surrounded by absorbing material (Eccosorb), leaving only the waveguide aperture exposed to the incident wave. The measurements were carried out using vertical polarization.

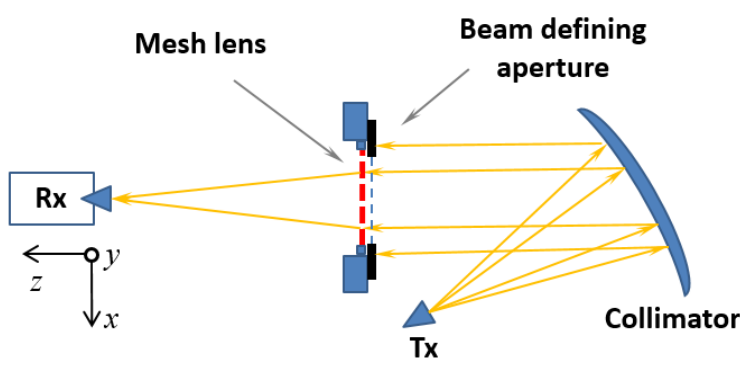

a)

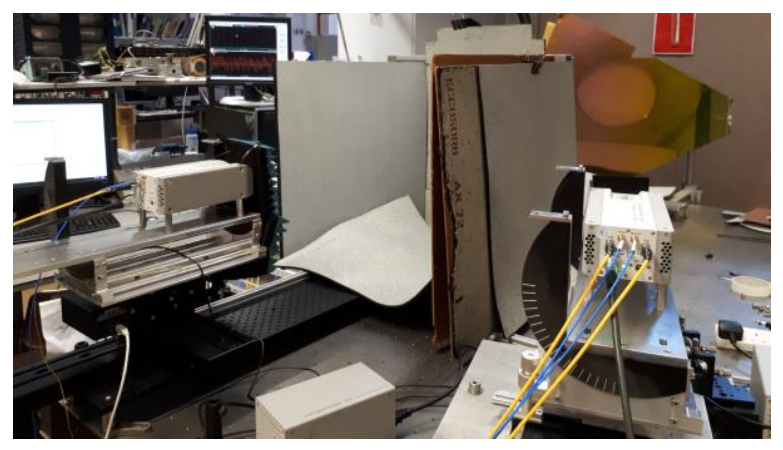

b)

Figure 11. a) Schematic of the near-field probing setup and (b) a view of the test bench showing the transmitter and receiver heads pointing towards each other through the beam collimator and the sample holder.

The transmitter horn was positioned at the focus of an off-axis mirror which transformed the generated spherical wavefront into a plane wave. This was then sent through a $288 \mathrm{~mm}$ circular metallic aperture surrounded by Eccosorb sheets. The lens was located just after the aperture and the receiver probe was able to move along the $x y$ and $x z$ planes, as shown in Fig. 11. This allowed planar longitudinal and transversal scans to be taken along the converging beam generated by the lens. Additional Eccosorb panels were used to avoid reflections from the bench and to avoid direct 
coupling of the transmitter horn with the lens and the probe. The mount was designed to allow the lens rotation around the system axis (z-axis in Fig. 11) and this proved to be useful for aligning the lens and to prove the rotational symmetry of the lens.

To evaluate the effective planarity of the collimated beam, transversal and longitudinal scans without the lens were taken at a distance from the aperture $z=1122 \mathrm{~mm}$, corresponding to the mesh lens focal distance at $125 \mathrm{GHz}$, see Fig. 12. The data shows little variation in amplitude across a transversal plane (of the order of $10^{-3}$ in signal magnitude) and almost flat and parallel phase fronts along a longitudinal cut. An additional test was carried out using a conventional polyethylene (PE) dielectric lens with similar focal length. Transversal scans were performed and compared with the theoretical expectations (Fig. 13). The good agreement between the PE lens Airy pattern and the ideal lens pattern confirmed the accuracy of the optical setup which was then ready for the mesh lens tests.

a)

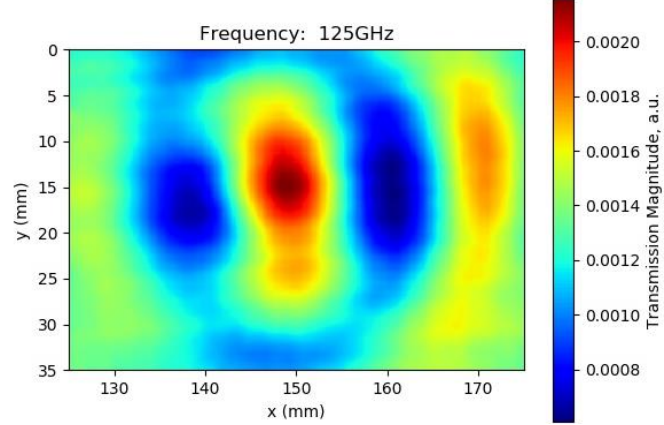

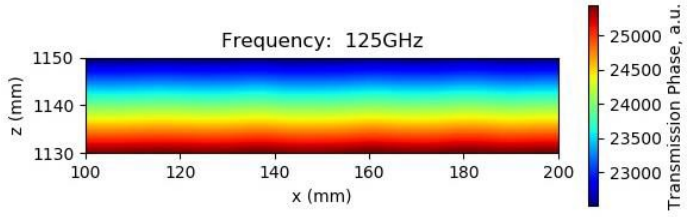

b)

Figure 12. a) Transversal cut of the magnitude and b) longitudinal cut of the phase of the plane wave transmitted through the empty $284 \mathrm{~mm}$ circular aperture, measured at the lens focal distance $z_{\mathrm{opt}}=1122 \mathrm{~mm}($ at $125 \mathrm{GHz})$.

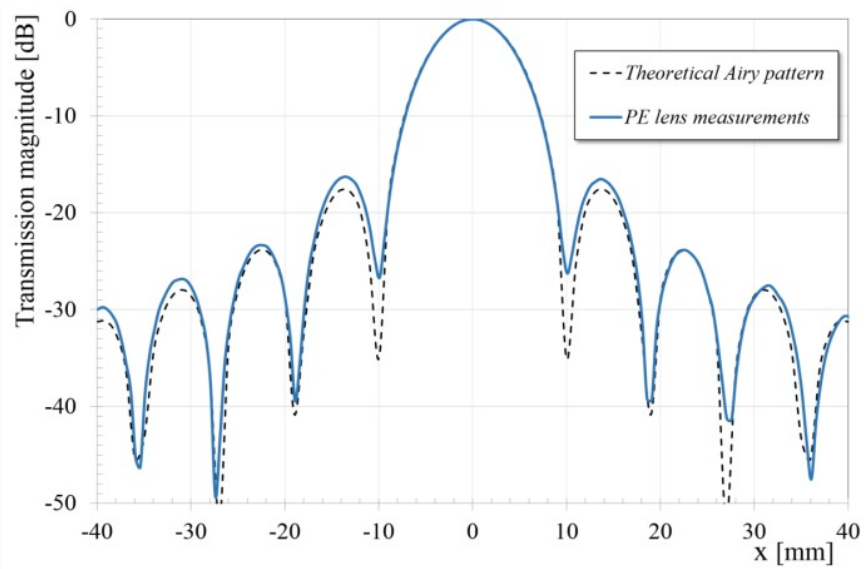

Figure 13. Check of the optical setup. Comparison between the measured focal plane Airy pattern produced by the test polyethylene lens (solid line) and the theoretical Airy pattern (dashed line). All measurements are shown at $125 \mathrm{GHz}$.

\subsection{Lens Characterization Results}

After locating the mesh lens behind the aperture, many longitudinal ( $x z$ - horizontal) and transversal ( $x y$ - vertical) scans were performed near the expected location of the lens focus. All the measurements were acquired within the frequency range 110 to $170 \mathrm{GHz}$. Example of results (at $110 \mathrm{GHz}$ ) are shown in Fig. 14. The xz-plane scan in Fig. 14a shows a pencil beam corresponding to the beam waist created by the lens around its focal point. This demonstrates the focusing capabilities of the lens. In the same figure the $x y$-plane transversal scan at the focal point is shown, which resembles the expected Airy pattern.

Additional measurements of the longitudinal cuts of the fields between 110 and $170 \mathrm{GHz}$ are reported in Fig. 15. The superimposed dashed line traces the position of the focal point as a function of frequency. The resulting lens focal length was found to be $f=1120 \pm 35 \mathrm{~mm}$. Considering that the effective tested diameter of the lens was $d_{e f f}=288 \mathrm{~mm}$, this leads 
to an f-number $f_{\# \#} \cong 3.9$ which is very close to the design target of $f / 4$. We notice that small anisotropic shrinkages of mesh-devices are normally observed after their fabrication and these could contribute to the observed small discrepancy in the f-number. We should also emphasize that the focal length variation $( \pm 35 \mathrm{~mm}$ within $110-170 \mathrm{GHz})$ indicates a relatively good achromatism of the lens, as compared to the alternative GrIn lens approach, discussed in Sec.2.

Fig. 16 shows transversal cuts measured between 120 and $170 \mathrm{GHz}$. These measurements prove the stability of the Airy pattern with frequency. The slight asymmetry in the amplitude of the first diffraction ring is probably created by the asymmetry of the optical setup, arising due to the off-axis mirror. We note also that these measurements were carried out using only one polarization state of the incident wave, the S-polarization.

We have compared the measured Airy patterns with the theoretical expectations. One example, at $110 \mathrm{GHz}$, is reported in Fig. 17. The measured FWHM of the mesh lens Airy pattern is $\sim 10.5 \mathrm{~mm}$ which is close to the $11.0 \mathrm{~mm}$ theoretical value. The slightly narrower pattern exhibits higher levels in the sidelobes, about $4 \mathrm{~dB}$ above that expected. This discrepancy could be attributed to the specific mesh lens design which, as mentioned earlier, showed a sudden jump in the pixel 'recipe' around 4/5 of the radius (see the pattern of the uncoated prototype in Fig. 8). The diffraction induced by this step in the overall pattern could be responsible for the higher level of sidelobes. Note that a discontinuity of the wavefront corresponding to that step was already visible in the finite-element simulations in Fig. 8.

To conclude, the mesh lens field pattern was modelled and fitted with a multi-mode Gaussian beam, which included the first six modes. The results of this fit are reported in Fig. 17.

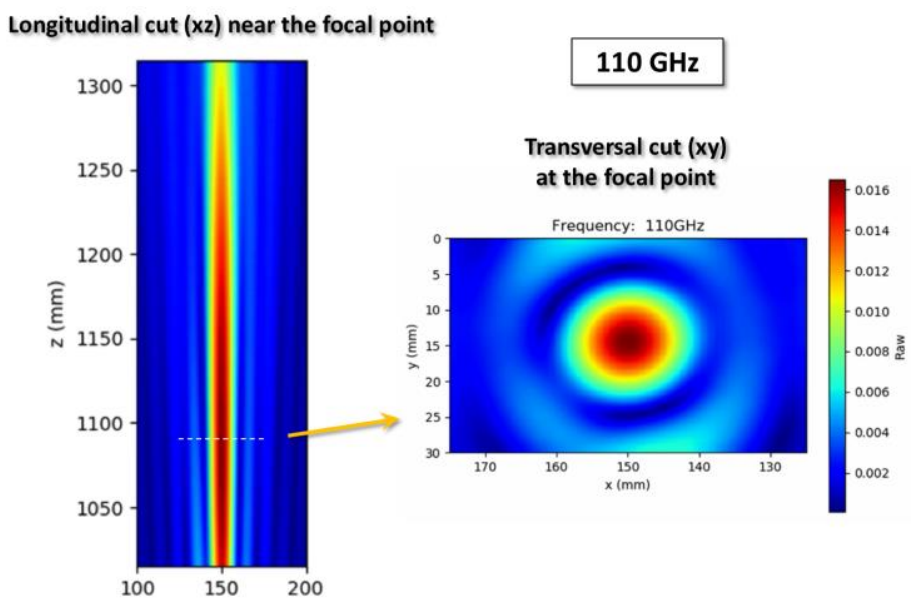

Figure 14. Example of measured longitudinal $(x z)$ and transversal $(x y)$ cuts of the electric field near the focus of the mesh lens. Measurements shown at $110 \mathrm{GHz}$.

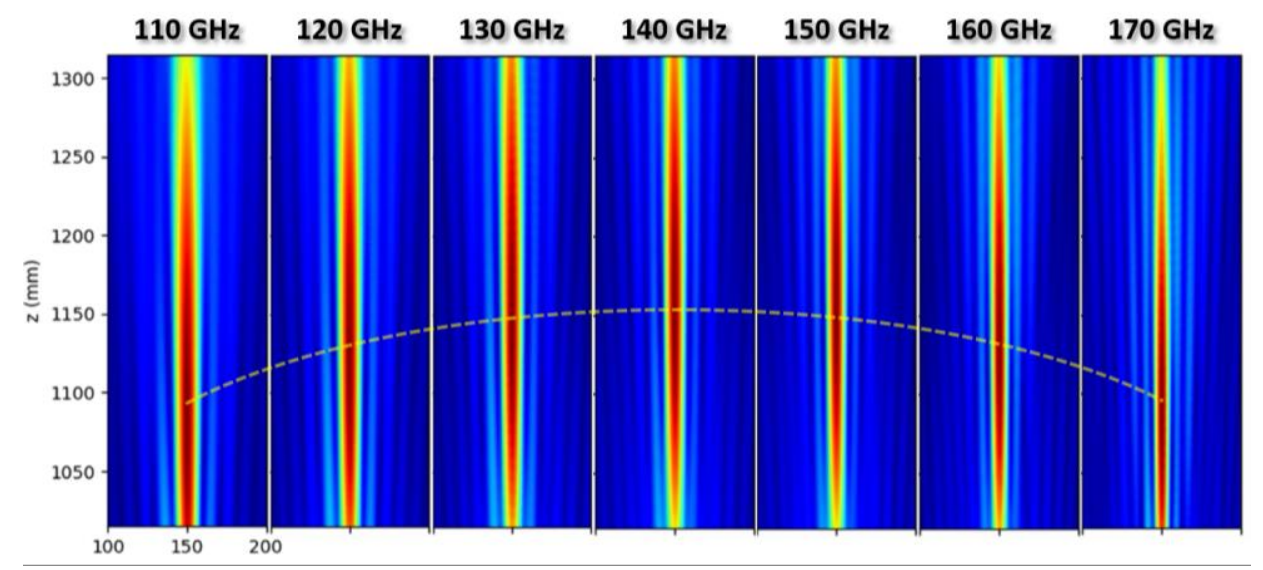

Figure 15. Measured longitudinal $x z$-cuts of the electric field near the focus of the mesh lens at different frequencies. The dashed line show the relatively small variation of the focal point with frequency. 

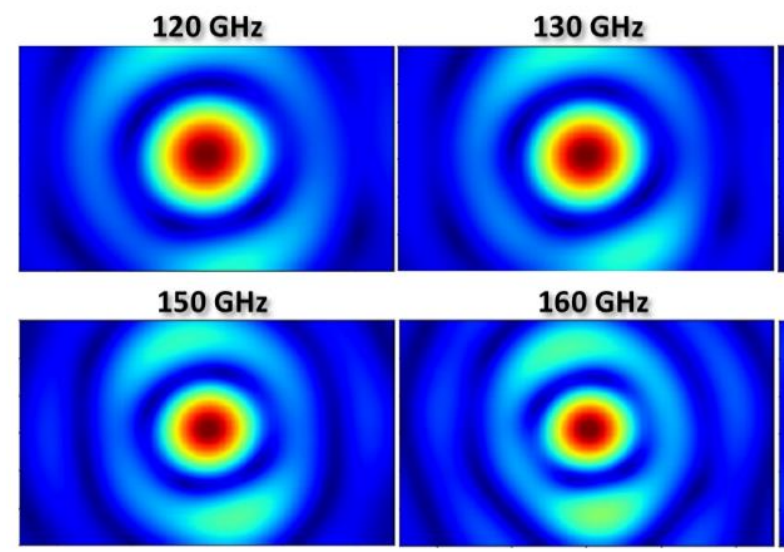

$160 \mathrm{GHz}$
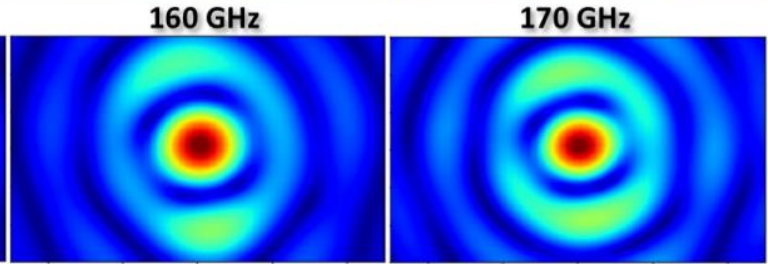

Figure 16. Transversal $x y$-cuts of the electric field near the focus of the mesh lens at different frequencies.

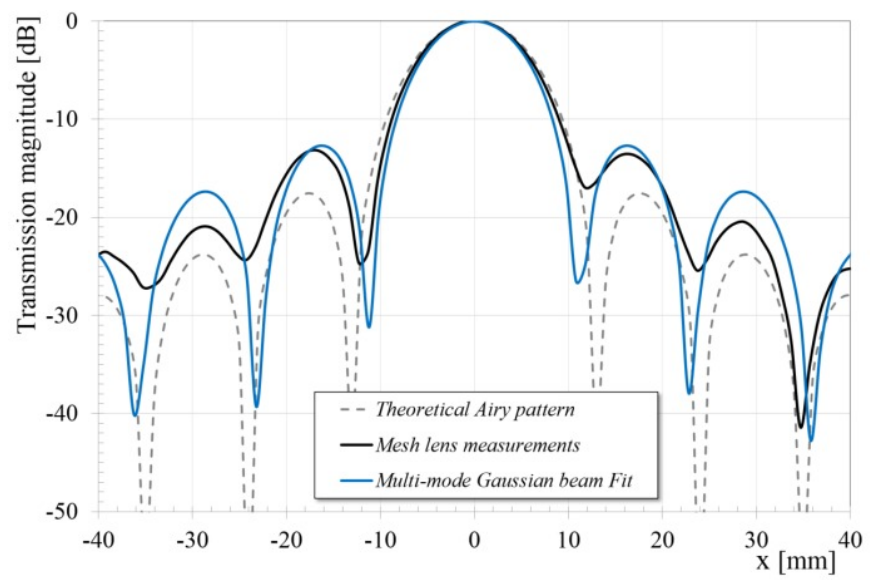

Figure 17. Airy pattern $x$-cut of the mesh lens (solid black) compared with the theoretical Airy pattern (dashed line) and the multi-mode Gaussian beam fit. All measurements and simulations at $110 \mathrm{GHz}$.

\section{CONCLUSIONS}

We have discussed the development of two types of large diameter flat metamaterial lenses: graded index and mesh lenses. Both solutions are based on the Cardiff metal-mesh filter technology and are designed to operate at millimeter wavelengths. New results of a previously realized $30 \mathrm{~cm}$ diameter graded index lens of the Fresnel type have been presented which shows that there is good agreement between the measured beam pattern and the ideal Airy fit. Further work is needed to investigate the addition of an anti-reflection coating to maximize the transmission.

For the first time we have demonstrated the design, manufacture and characterization of a large diameter $(30 \mathrm{~cm}) \mathrm{f} / 4$ mesh lens. Measurements of longitudinal and transversal cuts of the converging beam near the lens focus were recorded with a VNA and a 3D scanning system, within the 110-175 GHz frequency range. The results have confirmed the expected focusing capabilities of the mesh lens. The resulting focal length of the mesh lens are seen to show relatively little variation $(\sim 6 \%)$ across the band of investigation.

Further work would address a more detailed analysis of the mesh lens. This would include exploring the whole operational band of the lens $(75-175 \mathrm{GHz})$, a study of the polarization systematics and cross-polarization induced by the lens and the characterization of the lens using single-mode Gaussian beams without reflecting collimating mirrors. 


\section{ACKNOWLEDGMENTS}

This work was supported by the STFC Consolidated Grant ST/N000706/1 awarded at Cardiff University.

\section{REFERENCES}

[1] P. A. R. Ade, G. Pisano, C. E. Tucker and S. O. Weaver, "A review of metal mesh filters," Proceedings of SPIE: $6275,6275 \mathrm{U}$ (2006).

[2] G. Pisano, P. Ade, C. Tucker, P. Moseley and M.W. Ng, "Metal mesh based metamaterials for millimetre wave and THz astronomy applications," Proc. 8th UCMMT-2015 Workshop Cardiff, pp.1-4 (2016)

[3] Savini, Giorgio, Peter AR Ade, and Jin Zhang. "A new artificial material approach for flat THz frequency lenses." Optics express 20, no. 23 (2012): 25766-25773.

[4] G. Pisano, M.W Ng, B. Maffei and F.Ozturk, "A Dielectrically Embedded Flat Mesh Lens for Millimetre Waves Applications," Applied Optics, v. 52, n.11, pp.2218-2225 (2013),

[5] P. Moseley, G. Savini and P.r Ade. "Large Aperture Metal-Mesh Lenses for THz Astronomy" Proceedings EUCAP 2018

[6] G.Pisano, M.W. Ng, V. Haynes, B. Maffei, “A Broadband Metal-Mesh Half-Wave Plate for Millimetre Wave Linear Polarisation Rotation”, Progress In Electromagnetics Research M, 25, pp.101-114 (2012).

[7] G. Pisano, B. Maffei, P.A.R. Ade, P. de Bernardis, P. De Maagt, B. Ellison, M. Henry, M.W. Ng, B. Schortt, C. Tucker, "Multi-Octave Metamaterial Reflective Half-Wave Plate for Millimetre and Sub-Millimetre wave Applications," Applied Optics, 55, 10255-10262 (2016).

[8] Zhang, Jin, Peter AR Ade, Philip Mauskopf, Lorenzo Moncelsi, Giorgio Savini, and Nicola Whitehouse. "New artificial dielectric metamaterial and its application as a terahertz antireflection coating." Applied optics 48, no. 35 (2009): 6635-6642.

[9] Smith, David R., John B. Pendry, and Mike CK Wiltshire. "Metamaterials and negative refractive index." Science 305, no. 5685 (2004): 788-792.

[10] P. Moseley, G. Savini, E. Saenz, J. Zhang and P. Ade. "Detailed Characterization of a Lenster - A mm Flat Lens" IEEE Transactions on Antennas \& Propagation. Under Review

[11]P. Moseley, G. Savini, J. Zhang, and P. Ade. "Dual focus polarisation splitting lens." Optics Express 25, no. 21 (2017): 25363-25373.

[12] Ansys HFSS, www.ansys.com

Proc. of SPIE Vol. 10708 107080D-12 